\title{
Scalar gravitation: A laboratory for numerical relativity. III. Axisymmetry
}

\author{
Mark A. Scheel \\ Center for Radiophysics and Space Research and Department of Physics, Cornell University, Ithaca, New York 14853 \\ Stuart L. Shapiro and Saul A. Teukolsky \\ Center for Radiophysics and Space Research and Departments of Astronomy and Physics, Cornell University, Ithaca, New York 14853
}

(Received 15 September 1993)

\begin{abstract}
We construct a two-dimensional axisymmetric mean-field particle simulation scheme that solves the equations of relativistic scalar gravitation coupled to collisionless matter. Although scalar gravitation theory disagrees with experiment, it is useful as a testing ground for numerical methods used to solve the equations of general relativity, particularly in the generation of gravitational waves. We discuss methods for extracting the gravitational wave amplitude from the field variables, as well as methods for imposing an accurate outgoing-wave boundary condition on the scalar field at a finite radius. We find that for continuous matter distributions, our code is able to calculate smooth and accurate gravitational wave forms, despite the stochastic representation of the matter source terms caused by sampling with a finite number of particles. A similar scheme should provide accurate wave forms in general relativity, provided sufficient computer resources are used.
\end{abstract}

PACS number(s): 04.25.Dm, 02.70.Bf, 03.50.Kk, 04.30.Db

\section{INTRODUCTION}

Numerical codes for solving Einstein's equations of general relativity are generally quite complicated. This is especially true for those that simulate gravitational radiation, since this requires a nonspherical system involving at least two spatial dimensions plus time. Accordingly, it can be very difficult to develop and test new algorithms for numerical relativity: The human and computational resources required to simulate even the simplest dynamical problems can be considerable.

In order to study some of the computational aspects of numerical relativity in a simple setting, we examine a nonlinear scalar theory of relativistic gravitation that is less complicated than general relativity. This theory, which is presented in Ex. 7.1 of Ref. [1], contains some of the same features present in Einstein's theory, such as gravitational radiation and gravitational collapse, but without many of the complications, such as coordinate singularities and black holes. Of course, scalar gravitation fails miserably as a physical theory, disagreeing with the three classical experimental tests of general relativity. Nevertheless, it provides an ideal test site for developing the tools of numerical relativity.

We have previously $[2,3]$ discussed a numerical meanfield particle simulation scheme that solves the equations of scalar gravitation coupled to collisionless matter. (These papers will be referred to as papers I and II, and numbered equations from these papers will be prefixed by either I or II.) In paper I we examine spherical systems, exploiting the fact that scalar gravitational waves can be generated even in spherical symmetry. In Paper II, the scalar field is nonspherical but axisymmetric, and the matter is confined to an axisymmetric disk in the equatorial plane; in this way, it is possible to study the complications introduced by a two-dimensional gravitational field while retaining the simplicity of a one-dimensional matter distribution. Here we extend our treatment to general nonspherical matter configurations in axisymmetry. A key result of this paper is that it is possible to calculate smooth and accurate gravitational waveforms, despite the stochastic nature of the matter source terms in the gravitational field equation.

The fact that Einstein's equation is nonlinear, even in vacuum, complicates the problem of calculating gravitational radiation in numerical relativity. In scalar gravitation, the field equations are also nonlinear in the presence of matter, but they become linear in vacuum. This linearity allows us to determine the gravitational wave amplitude seen by an observer at infinity, given the scalar field as a function of time at an arbitrary finite radius outside the matter distribution. In addition, we use linearity to impose an outgoing-wave boundary condition on the scalar field at a finite radius - this condition remains accurate even if the coordinate grid is not much larger than the matter distribution itself.

\section{BASIC EQUATIONS}

The basic equations for relativistic scalar gravitation have already been presented in paper I, and will only be summarized here. We will adopt units where $c=1$, but we will display Newton's gravitational constant $G$ explicitly.

In scalar gravitation, particles move in Minkowski spacetime under the influence of a scalar field $\Phi$. The equation of motion for each particle takes the form

$$
\frac{d \widetilde{u}_{\mu}}{d \tau}=-e^{\Phi} \Phi_{, \mu}+\frac{1}{2} g_{\alpha \beta, \mu} e^{-\Phi} \widetilde{u}^{\alpha} \widetilde{u}^{\beta},
$$

where $u_{\mu}$ is a component of the particle's four-velocity, $\tau$ is its proper time, and 


$$
\tilde{u}_{\mu} \equiv e^{\Phi} u_{\mu}
$$

The spacetime is always Minkowskian and is not affected by the scalar field - the metric $g_{\mu \nu}$ appears in the above equation only to allow for curvilinear coordinates. In axisymmetry, we adopt spherical polar coordinates and set $x \equiv \cos \theta$. Eq. (2.1) takes the form

$$
\begin{aligned}
& \frac{d r}{d t}=\frac{\tilde{u}_{r}}{\tilde{u}^{0}}, \\
& \frac{d x}{d t}=-\frac{\tilde{u}_{\theta}}{\tilde{u}^{0} r^{2}}\left(1-x^{2}\right)^{1 / 2}, \\
& \frac{d \phi}{d t}=\frac{\tilde{u}_{\phi}}{\tilde{u}^{0} r^{2}\left(1-x^{2}\right)}, \\
& \frac{d \tilde{u}_{r}}{d t}=\frac{1}{\tilde{u}^{0} r^{3}}\left[\tilde{u}_{\theta}^{2}+\frac{\tilde{u}_{\phi}^{2}}{\left(1-x^{2}\right)}\right]-\frac{e^{2 \Phi} \Phi, r}{\tilde{u}^{0}}, \\
& \frac{d \tilde{u}_{\theta}}{d t}=\frac{x \tilde{u}_{\phi}^{2}}{r^{2}\left(1-x^{2}\right)^{3 / 2} \tilde{u}^{0}}+\frac{e^{2 \Phi} \Phi}{\tilde{u}^{0}}\left(1-x^{2}\right)^{1 / 2}, \\
& \frac{d \tilde{u}_{\phi}}{d t}=0,
\end{aligned}
$$

where

$$
\widetilde{u}^{0}=\left(e^{2 \Phi}+\tilde{u}_{r}^{2}+\frac{\widetilde{u}_{\theta}^{2}}{r^{2}}+\frac{\widetilde{u}_{\phi}^{2}}{r^{2}\left(1-x^{2}\right)}\right)^{1 / 2} .
$$

The angular momentum per unit mass of each particle, $\tilde{u}_{\phi}$, is strictly conserved.

The gravitational field evolves according to

$$
\square \Phi=4 \pi G e^{\Phi} \rho,
$$

where $\rho$ is the comoving density. Although the field equation is nonlinear in regions containing matter, it is linear in vacuum; this is not true in the case of general relativity. In axisymmetry, Eq. (2.5) becomes

$\Phi_{, t t}=\frac{1}{r^{2}}\left[r^{2} \Phi_{, r}\right]_{, r}+\frac{1}{r^{2}}\left[\left(1-x^{2}\right) \Phi_{, x}\right]_{, x}+4 \pi G T$,

where $T=-\rho e^{\Phi}$ is the trace of the matter stress-energy tensor.

If we assume reflection symmetry across the equatorial plane, we can solve Eq. (2.6) in the lower quadrant, subject to the boundary conditions

$$
\begin{aligned}
& \Phi_{, \theta}=-\left(1-x^{2}\right)^{1 / 2} \Phi_{, x}=0 \text { at } x=-1, \\
& \Phi_{, r}=0 \text { at } r=0, \\
& \Phi_{, x}=0 \text { at } x=0 .
\end{aligned}
$$

The first two of these equations are regularity constraints at the axis of symmetry and the origin; the third equation requires $\Phi$ to be equatorially symmetric. We also impose an outgoing-wave boundary condition at $r \rightarrow \infty$. In our numerical simulations this condition must be imposed at a finite value of $r$; the details of this will be discussed in Sec. IV D.

In two dimensions, the energy conservation law (I.2.19) remains valid. We write this relation in the form

$$
E(r, t)+S(r, t)=E(r, 0),
$$

where

$$
\begin{gathered}
E(r, t) \equiv \int_{0}^{r} r^{2} d r \int d \Omega\left[T^{00}-J^{0}\right] \\
=\int_{0}^{r} r^{2} d r \int d \Omega\left[\frac{1}{8 \pi G}\left(\Phi_{, 0}^{2}+(\nabla \Phi)^{2}\right]\right. \\
\left.+\rho \gamma\left(e^{\Phi} \gamma-1\right)\right], \\
S(r, t) \equiv r^{2} \int_{0}^{t} d t \int d \Omega\left[T^{0 r}-J^{r}\right] \\
=-r^{2} \int_{0}^{t} d t \int d \Omega\left[\frac{1}{4 \pi G} \Phi_{, 0} \Phi_{, r}\right. \\
\left.-\gamma \rho v_{r}\left(e^{\Phi} \gamma-1\right]\right] .
\end{gathered}
$$

The quantity $E$ is the total mass-energy within a sphere of radius $r$ centered at the origin, minus the total rest mass within the sphere. The quantity $S$ is the outgoing mass-energy flux through the sphere, minus the outgoing flux of rest mass through the sphere, integrated over time. It is useful to subtract the rest mass contributions to the energy and flux because our code, which deals with a fixed number of particles, automatically conserves rest mass.

For systems that settle into an equilibrium state, we find another check on our numerical integrations from the special relativistic virial theorem [Eqs. (I.2.20) and (I.2.21)]. If we define

$$
\begin{aligned}
& K=\frac{1}{2} \int \rho e^{\Phi} \gamma^{2} v^{2} d^{3} r, \\
& W=\frac{1}{2} \int \rho \Phi e^{\Phi} d^{3} r,
\end{aligned}
$$

then, in equilibrium,

$$
2 K=-W .
$$

In the Newtonian limit, $K$ and $W$ reduce to the usual Newtonian kinetic energy and gravitational potential energy.

When discussing gravitational waves, we concentrate on the variable

$$
\lambda \equiv \Phi_{, t}
$$

rather than on the variable $\Phi$, because $\Phi$ contains static Coulomb contributions as well as wavelike terms. In vacuum, $\lambda$ must satisfy

$$
\square \lambda=0,
$$

so in axisymmetry one may write

$$
\lambda(r, x, t)=\sum_{l=0}^{\infty} \lambda_{l}(r, t) P_{l}(x),
$$

where $\boldsymbol{P}_{l}$ is a Legendre polynomial. For an outgoing wave, $\lambda_{l}(r, t)$ takes the form [4] 


$$
\begin{aligned}
& \lambda_{0}(r, t)=\frac{F_{0}(t-r)}{r}, \\
& \lambda_{1}(r, t)=\frac{F_{1}^{\prime}(t-r)}{r}+\frac{F_{1}(t-r)}{r^{2}}, \\
& \lambda_{2}(r, t)=\frac{F_{2}^{\prime \prime}(t-r)}{r}+3 \frac{F_{2}^{\prime}(t-r)}{r^{2}}+3 \frac{F_{2}(t-r)}{r^{3}},
\end{aligned}
$$

for some set of functions $F_{l}$. Here primes denote derivatives with respect to the argument. Let $\lambda^{\mathrm{rad}}$ denote the radiative part of $\lambda$, that is, the part of $\lambda$ that falls off like $1 / r$. In spherical symmetry, only $\lambda_{0}$ can be nonzero, and therefore $\lambda=\lambda^{\mathrm{rad}}$ everywhere outside the matter. In general, equatorial symmetry allows $\lambda_{l}$ to be nonzero for any even $l$. Therefore $\lambda$ can contain not only radiative terms that fall off like $1 / r$, but also near-zone terms that fall off like higher powers of $1 / r$. As will be discussed in Sec. IV, we use Eq. (2.16) to extract $\lambda^{\text {rad }}$ from $\lambda(x, r, t)$, and also to impose the outgoing-wave boundary condition at a finite radius $r=r_{\text {max }}$.

\section{MULTIPOLE EXPANSION}

In the weak-field, slow-motion limit, one may obtain the radiation field by a multipole expansion. Using the usual Green's function for the scalar wave equation, we write Eq. (2.5) as

$$
\Phi(t, \mathbf{r})=-G \int d^{3} r^{\prime} \frac{1}{R}\left[\frac{e^{\Phi} \rho_{0}}{\gamma}\right]_{\mathrm{ret}},
$$

where $R=\left|\mathbf{r}-\mathbf{r}^{\prime}\right|, \rho_{0}$ is the rest mass density, $\gamma$ is the usual Lorentz factor, and "ret" means evaluate at the retarded time $t^{\prime}=t-R$. We first expand $\rho_{0}$ and $e^{\Phi} / \gamma$ :

$$
\begin{array}{r}
\rho_{0}\left(t-R, \mathbf{r}^{\prime}\right)=\rho_{0}\left(t-r, \mathbf{r}^{\prime}\right)+(r-R) \rho_{0, t} \\
+\frac{1}{2}(r-R)^{2} \rho_{0, t t}+\cdots, \\
\frac{e^{\Phi}}{\gamma}=\left[1+\Phi-\frac{1}{2} v^{2}\right]_{t^{\prime}=t-r}+\cdots,
\end{array}
$$

and we obtain

$$
\Phi(t, \mathbf{r}) \approx-G \int d^{3} r^{\prime} \frac{1}{R}\left[\rho_{0}\left(1+\Phi-\frac{1}{2} v^{2}\right)+\rho_{0, t}(r-R)+\frac{1}{2}(r-R)^{2} \rho_{0, t t}+\cdots\right]_{t-r}
$$

Now we expand the factors involving $R$ in powers of $r^{\prime} / r$ :

$$
\begin{aligned}
& \frac{1}{R}=\frac{1}{r}\left[1+u\left[\frac{r^{\prime}}{r}\right]+\frac{1}{2}\left(3 u^{2}-1\right)\left(\frac{r^{\prime}}{r}\right)^{2}+\cdots\right], \\
& \frac{r-R}{R}=u\left[\frac{r^{\prime}}{r}\right]+\frac{1}{2}\left(3 u^{2}-1\right)\left[\frac{r^{\prime}}{r}\right]^{2}+\cdots, \\
& \frac{(r-R)^{2}}{R}=r\left[u^{2}\left[\frac{r^{\prime}}{r}\right]^{2}+\left(2 u^{3}-u\right)\left(\frac{r^{\prime}}{r}\right)^{3}+\cdots\right],
\end{aligned}
$$

where

$$
u \equiv \frac{\mathbf{r} \cdot \mathbf{r}^{\prime}}{r r^{\prime}} .
$$

If $\rho_{0}$ is axially and equatorially symmetric, then all terms in Eq. (3.5) containing odd powers of $u$ will integrate to zero when inserted into Eq. (3.4).

In the wave zone $\left(r \gg r^{\prime}\right)$, we keep only the terms in Eq. (3.5) that fall off like $1 / r$. The leading-order term, which involves the product of $\rho_{0}$ from Eq. (3.2) with the 1 from Eq. (3.3) and the $1 / r$ from Eq. (3.5a), gives $M_{0} / r$, where $M_{0}$ is the conserved rest mass. This term does not contribute to the radiation field because it is constant in time, so it will be dropped. The remaining terms give us $\Phi^{\mathrm{rad}}$, the radiative part of the gravitational field. From Eq. (3.4) we obtain

$$
-r \Phi^{\mathrm{rad}}(t, \mathbf{r}) \approx 4 \pi G \int_{0}^{\infty} r^{\prime 2} d r^{\prime} \int_{-1}^{0} d x^{\prime}\left[\rho_{0}\left(\Phi-\frac{v^{2}}{2}\right)+\frac{\rho_{0, t t}}{3}\left[P_{2}(x) P_{2}\left(x^{\prime}\right)+\frac{1}{2}\right]\right]_{t-r},
$$

where $x=\cos \theta, x^{\prime}=\cos \theta^{\prime}$, and $P_{2}$ is a Legendre polynomial. Here we have assumed axial and equatorial symmetry, and we have performed the integration over $\phi^{\prime}$. The gravitational wave amplitude $\lambda^{\text {rad }}$ is obtained by taking the time derivative of Eq. (3.6).
As one can see from Eq. (3.6), the dominant radiation field is a superposition of monopole and quadrupole terms; some of the terms we have neglected contain higher angular moments. The neglected terms are smaller than the dominant terms either by powers of $r^{\prime} / r$ from 
Eq. (3.5), by powers of $\Phi$ from Eq. (3.3), or by powers of $v$ from either Eq. (3.3) or from higher-order time derivatives of $\rho_{0}$ in Eq. (3.2). Notice that there exist near-zone terms $\lambda^{\mathrm{NZ}}$ that fall off as $1 / r^{2}, 1 / r^{3}$, etc., as well as the $1 / r$ radiation term $\lambda^{\mathrm{rad}}$. The largest of these near-zone terms comes from the $1 / r^{2}$ part of the $\rho_{0, t}$ term in Eq. (3.4). This term is purely quadrupole, and for $r=r^{\prime}$ it is larger than the leading-order quadrupole radiation term by a factor of $1 / v$. Because of this, we cannot blindly assume that the near-zone terms are negligible when observing $\lambda$ at a finite radius $r$.

\section{NUMERICAL METHOD}

As described in papers I and II, we use a mean-field particle simulation scheme to solve the coupled equations of motion for the particles and the scalar field: During each time step of length $\Delta t$, the source term $T$ appearing in Eq. (2.6) is calculated from the positions and velocities of the particles. The particles are then moved according to the mean field $\Phi$. Finally, the field is evolved for a time $\Delta t$ according to Eq. (2.6), and the process is repeated.

\section{A. Equations of motion}

As in papers I and II, the field equation (2.6) is solved by an explicit leapfrog method with a variable time step. When written in terms of the variables $\Phi$ and $\lambda$, the second-order field equation becomes two coupled firstorder equations:

$$
\begin{aligned}
& \mathcal{T}[\Phi]=\lambda, \\
& \mathcal{T}[\lambda]=\mathscr{R}[\Phi]+\mathscr{F}[\Phi]+4 \pi G T,
\end{aligned}
$$

where the derivative operators $\mathcal{T}, \mathcal{R}$, and $\mathcal{F}$ are given by Eq. (II.4.3).

To finite difference these equations, we introduce a two-dimensional grid $\left\{r_{i}, x_{k}\right\}$, defined as in paper II. The quantities $\Phi$ and $\lambda$ are defined at the midpoint of each grid zone. The radial grid is laid out as in paper $I$, and the angular grid is uniform in $x$. The finite-difference forms of $\mathcal{T}, \mathscr{R}$, and $\mathcal{F}$ are given by Eq. (II.4.4).

As in paper II, we need not explicitly impose boundary conditions at the origin or on the axis. At the equator we impose reflection symmetry:

$Y_{i+1 / 2, k+1 / 2}^{n}-Y_{i+1 / 2, k-1 / 2}^{n}=0$ at $k=k_{\max }$,

where $Y$ denotes either $\Phi$ or $\lambda$. At the outer grid boundary, $r=r_{i_{\max }}=r_{\max }$, we do not need to impose a boundary condition on $\Phi$ because no spatial derivatives appear in Eq. (4.1a). However, we must impose a boundary condition on $\lambda$ at $r=r_{\max }$ : we choose an outgoing-wave condition that will be discussed in Sec. IV D.

For numerical stability, we put an upper bound on the time step according to Eq. (II.4.8). We find empirically that this criterion yields a stable scheme even when the grid is adjusted to follow the matter on each time step. To prevent the growth of high frequency instabilities associated with the leapfrog scheme, we add a small
Kreiss-Oliger [5] diffusive term to the evolution equations (4.1).

The source term $T$ appearing in Eq. (4.1) is evaluated by summing over the particles in a particular zone:

$T=-\sum m_{A} n_{A} e^{\Phi}=-\sum m_{A}\left(n_{A} \gamma_{A}\right) \frac{1}{\tilde{u}_{A}^{0}} e^{2 \Phi}$

where $m_{A}$ is the rest mass of particle $A$, and the comoving number density $n_{A}=\rho_{A} / m_{A}$ is given by

$$
n_{A}=\frac{1}{\mathcal{V}_{A}}=\frac{1}{2 \pi \gamma_{A} \Delta\left(r^{3} / 3\right) \Delta x} \text {. }
$$

Here $\mathcal{V}_{A}$ is the comoving volume for particle $A$-we treat the particle as smeared out over the entire zone. To evaluate the energy and flux integrals in Eqs. (2.9) and (2.10), we also need two other matter source termsthese are given by Eq. (I.5.12).

In order to obtain a smooth density profile from a finite number of particles, we use a two-dimensional generalization of the Hockney-Eastwood algorithm (Eqs. 5-88 of Ref. [6]) to share each particle's rest mass among its own grid zone and each of the eight neighboring zones: First, the particle's mass is distributed among the three nearest radial zones according to the Hockney-Eastwood scheme; if the particle occupies the first or last radial zone, the contribution that would have been assigned to the nonexistent adjacent zone is assigned instead to the particle's own radial zone. Next, the contribution from each of these radial zones is distributed among the three nearest angular zones; again, any contribution that would have been assigned to a nonexistent zone is assigned instead to the particle's own angular zone. Finally, we multiply the fraction of each particle's mass that is to be assigned to a certain zone by the volume of that zone, and we renormalize to conserve rest mass. This procedure ensures that a homogeneous particle distribution yields a homogeneous density profile even when adjacent zones have unequal volume.

The particle equations of motion (2.3) are evolved by an embedded fourth-fifth order Runge-Kutta scheme with adaptive step size [7]. The components of $\nabla \Phi$ in Eq. (2.3) are obtained by finite differencing $\Phi$ and then interpolating to the particle position. Particles that attempt to cross the equatorial plane are reflected back into the correct quadrant; this is equivalent to assuming that any particle crossing from the lower to the upper quadrant has a counterpart with $\tilde{u}_{r}^{\text {new }}=\tilde{u}_{r}, \tilde{u}_{\theta}^{\text {new }}=-\widetilde{u}_{\theta}$ crossing the other way. A particle with $\widetilde{u}_{\phi}=0$ that attempts to cross the symmetry axis is likewise replaced by a counterpart with $\tilde{u}_{r}^{\text {new }}=\tilde{u}_{r}, \tilde{u}_{\theta}^{\text {new }}=-\widetilde{u}_{\theta}$.

\section{B. Multipole expansion}

For weak fields and small velocities, we may use the multipole expansion formula (3.6) to test the accuracy of our code. For a distribution of point particles labeled by the index $A$, we can use the identity (I.5.14) to write Eq. (3.6) in the form 


$$
\begin{aligned}
-r \Phi(t, \mathbf{r})^{\mathrm{rad}} \approx G \sum_{A} m_{A} & {\left[\Phi-\frac{v_{A}^{2}}{2}+\frac{1}{4}\left(r_{A}^{2} x_{A}^{2}\right]_{, t t}\left(3 x^{2}-1\right)\right.} \\
& \left.+\frac{1}{4}\left[r_{A}^{2}\right]_{, t t}\left(1-x^{2}\right)\right]_{t-r}
\end{aligned}
$$

Evaluating the time derivatives using Eq. (2.3), we obtain

$$
-r \Phi(t, \mathbf{r})^{\mathrm{rad}} \approx G \sum_{A} m_{A}\left[\alpha_{A}+\beta_{A} P_{2}(x)\right]_{t-r},
$$

where, for each particle,

$$
\begin{aligned}
\alpha= & \Phi-\frac{\tilde{u}_{r}^{2}}{6}-\frac{\tilde{u}_{\theta}^{2}}{6 r^{2}}-\frac{\tilde{u}_{\phi}^{2}}{6 r^{2}\left(1-x^{2}\right)}-\frac{r \Phi_{, r}}{3}, \\
3 \beta= & \left(3 x^{2}-1\right) \tilde{u}_{r}^{2}+\left(2-3 x^{2}\right) \frac{\tilde{u}_{\theta}^{2}}{r^{2}}-\frac{\tilde{u}_{\phi}^{2}}{r^{2}\left(1-x^{2}\right)} \\
& -\frac{6 \tilde{u}_{\theta} \tilde{u}_{r} x \sqrt{1-x^{2}}}{r}+\left(1-3 x^{2}\right) r \Phi_{, r}-3 x\left(1-x^{2}\right) \Phi_{, x} .
\end{aligned}
$$

In Eq. (4.7), $\Phi$ and its derivatives are to be evaluated at the particle position. To obtain the radiative wave amplitude $\lambda^{\mathrm{rad}}$, we take a numerical time derivative of Eq. (4.6).

\section{Wave extraction}

The appropriate variable used to describe the gravitational radiation produced by a localized matter distribution is $\lambda^{\mathrm{rad}}$, the radiative $1 / r$ term of $\lambda$. If $r$ is not sufficiently large, the nonradiative near-zone terms $\lambda^{\mathrm{NZ}}$ may be comparable in magnitude to $\lambda^{\mathrm{rad}}$. Therefore, the variable $\lambda(r)$ is not the radiation field of interest unless $r$ is large. One encounters a similar difficulty in general relativity, but there the problem is compounded by the fact that the field equation is nonlinear even in vacuum, and that there may exist gauge (coordinate) waves as well as physical gravitational waves.

We have examined two solutions to this problem. The first is simply to put the observation point at a sufficiently large radius so that the near-zone terms are negligible. This "brute force" approach will work, but it requires one to increase the outer grid radius $r_{\max }$ and the number of radial grid zones covering the volume within $r=r_{\max }$. In addition, the system must be evolved for a larger number of time steps in order to allow the wave to propagate to the observation point. This solution can therefore be costly in terms of computer time.

The second solution is to take advantage of the fact that in vacuum, the field equation is linear and must obey Eqs. (2.15) and (2.16). From the known value of $\lambda(r, x, t)$, we find $\lambda_{l}(r, t)$ by inverting Eq. (2.15):

$$
\lambda_{l}(r, t)=(2 l+1) \int_{-1}^{1} \lambda(r, x, t) P_{l}(x) d x .
$$

For equatorial symmetry, $\lambda_{l}(r, t)$ is zero for odd $l$. At a fixed value of $r$, we integrate the ordinary differential equations (2.16) with respect to time, and thus obtain the functions $F_{l}(t-r)$. Once these functions are known, we can reconstruct the radiative part of the wave:

$$
\begin{aligned}
r \lambda^{\mathrm{rad}}(r, t)= & F_{0}(t-r)+F_{1}^{\prime}(t-r) P_{1}(x) \\
& +F_{2}^{\prime \prime}(t-r) P_{2}(x)+\cdots .
\end{aligned}
$$

This method of wave extraction was originally introduced in general relativity by Abrahams and Evans [8]. In the general relativistic case, the method can only be used far from the source, where the linearized Einstein equations are valid. In scalar gravitation, however, wave extraction can be performed at any radius outside the matter, since the vacuum field equations are linear. We only evaluate Eq. (4.9) for $l=0$ and $l=2$, since the terms with $l>2$ are small. We find that for $l \leq 2$, it is sufficient to integrate Eq. (2.16) by an explicit Euler method, using the current value of $\lambda(r, x, t)$ and one previously saved value.

\section{Outgoing-wave boundary condition}

In general, it can be difficult to construct a boundary condition at a finite radius that allows arbitrary outgoing waves to pass without reflection. If the system is spherically symmetric, or if the outer grid boundary $r_{\max }$ is large enough that the near-zone contributions to $\lambda$ are negligible there, then the boundary condition used in papers $I$ and II is sufficient:

$$
(r \lambda)_{, t}+(r \lambda)_{, r}=0 \text { at } r=r_{\max } .
$$

However, if one attempts to use this boundary condition for a nonspherical case in which $\lambda \neq \lambda^{\mathrm{rad}}$ at $r=r_{\max }$, any terms in $\lambda$ that fall off like $1 / r^{2}$ or higher powers of $1 / r$ will be partially reflected off the outer grid boundary.

In scalar gravitation, we can use the fact that the field equation is linear in vacuum, so $\lambda$ must satisfy Eqs. (2.15) and (2.16). From Eq. (2.16), we construct a boundary condition analogous to Eq. (4.10):

$$
(r \lambda)_{, r}+(r \lambda)_{, t}=R \text {, }
$$

where

$$
\begin{aligned}
R(r, x, t) \equiv & P_{1}(x)\left[\frac{-F_{1}(t-r)}{r^{2}}\right] \\
& +P_{2}(x)\left[-3 \frac{F_{2}^{\prime}(t-r)}{r^{2}}-6 \frac{F_{2}(t-r)}{r^{3}}\right]+\cdots
\end{aligned}
$$

In equatorial symmetry, the coefficients of the odd Legendre polynomials vanish. For spherical symmetry or for large $r$, Eq. (4.11) reduces to Eq. (4.10). A second-order accurate finite difference form of Eq. (4.11) is

$$
\begin{aligned}
\lambda_{i+1 / 2, k+1 / 2}^{n+1}= & \chi \lambda_{i-1 / 2, k+1 / 2}^{n} \\
+ & {\left[\frac{1-\zeta}{1+\zeta}\right]\left[\lambda_{i+1 / 2, k+1 / 2}^{n}-\chi \lambda_{i-1 / 2, k+1 / 2}^{n+1}\right) } \\
& +\frac{2 \Delta t_{n}}{r_{i+1 / 2}(1+\zeta)} R_{i, k}^{n+1 / 2}
\end{aligned}
$$

where 


$$
\begin{aligned}
& \zeta \equiv \frac{\Delta t_{n}}{r_{i+1 / 2}-r_{i-1 / 2}}, \\
& \chi \equiv \frac{r_{i-1 / 2}}{r_{i+1 / 2}} .
\end{aligned}
$$

To impose the outgoing-wave boundary condition (4.11) we proceed as follows: We first choose a radius $r_{0}$ at which to extract the wave amplitude. At each time step, we extract the quantities $F_{l}\left(t-r_{0}\right)$ from $\lambda$ according to the procedure of Sec. IV C, and store the values of $t$ and $F_{l}\left(t-r_{0}\right)$ in a table. To evaluate the quantity $R\left(r_{\max }, x, t_{0}\right)$ at time $t_{0}$ and radius $r_{\max }$, we search the table for the two entries containing times $t$ nearest to the retarded time $t_{\text {ret }}=t_{0}-\left(r_{\max }-r_{0}\right)$. We then interpolate between these entries to obtain $F_{l}\left(t_{\text {ret }}-r_{0}\right)$, which is equal to $F_{l}\left(t_{0}-r_{\max }\right)$, and we evaluate $R\left(r_{\max }, x, t_{0}\right)$ from Eq. (4.12). Finally, we impose the outgoing-wave boundary condition by using Eq. (4.13) to set the value of $\lambda_{i+1 / 2, k+1 / 2}^{n+1}$ at $i=i_{\max }$. We typically choose $r_{0}=0.9 r_{\max }$. Empirically, we find that the final result is insensitive to this choice. Since we only keep terms with $l \leq 2$ in our wave extraction (4.9), we do not include any terms with $l>2$ when evaluating Eq. (4.12).

\section{E. Matter distribution}

Several of the matter configurations studied in papers I and II possessed homogeneous density profiles. Homogeneity is useful because it often allows one to find simple analytic solutions. However, a homogeneous matter distribution has a sharp discontinuity at the matter surface. For a two-dimensional system, this discontinuity introduces serious difficulties for finite-difference methods.

Consider what happens when a sharp matter surface crosses a grid zone. At each time step, the contribution to the new value of $\lambda$ at a specific grid point $\{i+1 / 2, k+1 / 2\}$ due to the source term $T$ is

$$
\frac{\Delta t_{n}}{\Delta t_{n-1}}\left(\Delta t_{n}+\Delta t_{n-1}\right) 4 \pi G T,
$$

as calculated from Eqs. (4.1b) and (II.4.4). Suppose that during the time step $\Delta t_{n}$, the matter surface has crossed the grid point $\{i+1 / 2, k+1 / 2\}$, so that the source term has changed discontinuously from $T$ to zero or vice versa. Assuming that the values of $\lambda$ and $\Phi$ at other grid points have not changed much during this time step (which must be the case if any finite-difference method is to be accurate), the value of $\lambda$ at $\{i+1 / 2, k+1 / 2\}$ will suddenly change by an amount given in Eq. (4.15). For typical values of $\Delta t_{n}, \Delta t_{n-1}$, and $T$, this change can be on the order of $\lambda$ itself. When such a discontinuity arises, it becomes amplified by subsequent finite differencing and propagates through the grid, destroying the accuracy of the simulation.

Note that in the limit $\Delta t_{n} \rightarrow 0$, the expression (4.15) vanishes; hence one solution to the problem is to decrease the time step. However, this is very costly in terms of computer time-we would most likely need to decrease the time step by more than an order of magnitude before the problem disappeared. Another solution is to move the grid on every time step so that the sharp matter surface never crosses a grid zone-this is what was done in papers I and II, so that the matter discontinuity in the radial direction caused no difficulty whatsoever. (In paper II, the matter was confined to a thin disk, and the discontinuity in the angular direction was handled by a jump condition; this was possible because the disk resided at $x=0$ for all times.) Unfortunately, this is difficult to accomplish for an arbitrary two-dimensional matter distribution without drastically modifying the code, e.g., rejecting spherical polar coordinates in favor of a coordinate system that can vary arbitrarily with the matter.

There is also the possibility that the smoothing algorithm for the matter source terms described in Sec. IV A could be used to smooth the discontinuity at the matter surface. However, for a true homogeneous density profile, one would expect the discontinuity to contribute significantly to the gravitational radiation (i.e., the quantities $\rho_{0, t}$ and $\rho_{0, t t}$ in Eq. (3.4) include terms involving $\delta$ functions), so such smoothing would in fact be artificial and undesirable.

We therefore choose to avoid the problem altogether by using an initial density profile that tends to zero smoothly at the matter surface, and by limiting ourselves to cases in which $\rho$ remains continuous at all times.

\section{F. Initial data}

All of the problems we have analyzed start from a moment of time symmetry, so that

$$
\begin{aligned}
& \lambda=0, \\
& \nabla^{2} \Phi=4 \pi G e^{\Phi} \rho,
\end{aligned}
$$

subject to the boundary condition

$$
(r \Phi)_{, r}=0 \text { at } r \rightarrow \infty,
$$

as well as the boundary conditions (2.7). These equations are solved by iteration starting with an initial guess for $\Phi$ on the right-hand side of Eq. (4.16b). Finite differencing using the operators $\mathcal{R}$ and $\mathcal{F}$ in Eq. (II.4.4) yields a sparse system of linear equations that are iterated to convergence.

We choose a random sample of particles to represent the given initial density and velocity profiles of the matter. For sufficient resources, this scheme yields initial data of arbitrary accuracy even for strong fields and large particle velocities.

\section{NUMERICAL RESULTS}

When analyzing our numerical results, we will adopt gravitational units where $G=c=1$.

\section{A. Radiation from analytic matter distribution}

In order to calibrate the wave propagation properties of our numerical code without the complications introduced by a discrete particle distribution, we construct cases in which the matter source $\rho(r, x, t)$ is given explicitly by an analytic expression. In addition, we also simplify the field equation by setting $e^{\Phi}=1$ in the source term $T$ 
and setting $\rho=\rho_{0}$. Employing an analytic matter source is useful for determining how well our code solves Eq. (2.5) with various grid choices and outgoing-wave boundary conditions.

The analytic matter distribution is at all times taken to be spheroidal and axisymmetric. Let $a$ denote the radial coordinate of the surface of the spheroid in the equatorial plane, and let $c$ denote the radial coordinate of the surface along the symmetry axis. Hence the interior of the spheroid is the region

$$
\frac{r^{2} x^{2}}{c^{2}}+\frac{r^{2}\left(1-x^{2}\right)}{a^{2}}<1,
$$

where we have used the spherical polar coordinates $r$ and $x$. If $a>c$ the spheroid is oblate, and if $a<c$ it is prolate. The density profile is chosen as

$$
\rho=\frac{105}{9} \frac{M}{4 \pi a^{2} c}\left(1-\frac{r^{2}\left(1-x^{2}\right)}{a^{2}}-\frac{r^{2} x^{2}}{c^{2}}\right)^{2} .
$$

The function $\rho(r, x, t)$ has the property that both it and its normal gradient vanish at the surface of the spheroid.

Provided $a$ and $c$ are large $\left(\gg M_{0}\right)$ and their time derivatives are small $(<<1)$, we can obtain the wave amplitude by integrating the multipole expansion formula (3.4) analytically, keeping terms up to order $\left(r^{\prime} / r\right)^{2}$ in Eqs. (3.5). Since we have also set $\gamma=e^{\Phi}=1$ in the field equation, we must omit the $\Phi-v^{2} / 2$ that multiplies the $\rho_{0}$ in Eq. (3.4). For $\rho(r, x, t)$ given by Eq. (5.2), we obtain, at any fixed radius,

$$
\begin{aligned}
-9 r \lambda(t) & =\frac{1}{3}(\dddot{c} \dot{c}+3 \dddot{c} \dot{c})+\frac{2}{3}(\dddot{a} a+3 \dddot{a} \dot{a}) \\
& +\frac{2 P_{2}(x)}{3}(\dddot{c} \dot{c}+3 \dddot{c} \dot{c}-\dddot{a} a-3 \ddot{a} \dot{a}) \\
& +\frac{2 P_{2}(x)}{r}\left(\ddot{c} c+\dot{c}^{2}-\ddot{a} a-\dot{a}^{2}\right) \\
& +\frac{2 P_{2}(x)}{r^{2}}(\dot{c} c-\dot{a} a),
\end{aligned}
$$

where dots denote time derivatives. The first three terms in Eq. (5.3) give $\lambda^{\mathrm{rad}}$, the radiative part of the wave amplitude $\lambda$, while the other two terms represent $\lambda^{\mathrm{NZ}}$, the leading-order near-zone contribution to $\lambda$. For large $r$, $\lambda^{\mathrm{rad}} \gg \lambda^{\mathrm{NZ}}$. However, $\lambda^{\mathrm{rad}}$ contains more time derivatives than $\lambda^{\mathrm{NZ}}$, so if $r$ is not very large, $\lambda^{\mathrm{rad}}$ will be smaller than $\lambda^{\mathrm{NZ}}$ by factors of $v$.

Figure 1 shows the wave amplitude $\lambda$, multiplied by $r$, for a case in which an initially spherical configuration of radius $50 M_{0}$ is periodically compressed along the symmetry axis:

$$
\begin{aligned}
& a(t)=50 M_{0}=\text { const }, \\
& c(t)=45 M_{0}+5 M_{0} \cos \omega t .
\end{aligned}
$$

The oscillation frequency is chosen to be $\omega=6.61 \times 10^{-3} M_{0}^{-1}$. For this case we use 90 exterior and 90 interior radial zones, and 16 angular zones. At $r=r_{\max }=200 M_{0}$ we impose the simple outgoing-wave boundary condition (4.10). Notice that our numerical
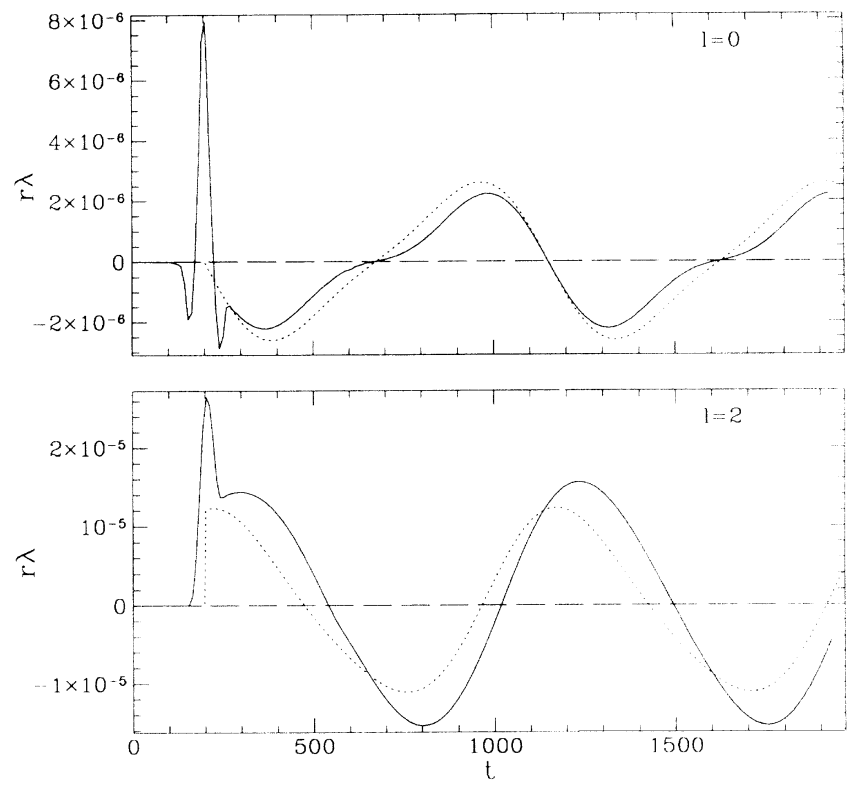

FIG. 1. First two nonvanishing angular moments of the wave amplitude $\lambda$ (multiplied by $r$ ) as a function of time, measured at $r=200 M_{0}$ for an analytic $\rho(r, x)$. The solid line is the numerical value of $\lambda$ obtained from Eq. (4.1) with outgoing-wave boundary condition (4.10), and the dotted line is the analytic result, Eq. (5.3). The bumps near $t=200$ are due to a transient that results from starting the numerical integration at $t=0$ rather than at $t=-\infty$. The quantity $r \lambda$ is dimensionless, while $t$ is in units of $M_{0}$. Note the difference in scale between the top and bottom plots.

wave amplitude $\lambda$ does not agree very well with the analytic result (5.3); this is mainly due to reflections off the outer grid boundary: The boundary condition (4.10) is only adequate for $\lambda \sim 1 / r$, while in this case the magnitude of $\lambda^{\mathrm{NZ}}$ is comparable to that of $\lambda^{\mathrm{rad}}$, even at $r=200$ $M_{0}$.

In Fig. 2, we show the wave amplitude $\lambda$ for a situation identical to the one shown in Fig. 1, except that at $r=r_{\max }$ we impose Eq. (4.11) rather than Eq. (4.10). The quadrupole component of $\lambda$ now agrees with the analytic result-Eq. (4.11) prevents the $1 / r^{2}$ and $1 / r^{3}$ terms of $\lambda_{l=2}$ from reflecting off the grid boundary at $r=r_{\max }$. Notice that $\lambda_{l=0}$ does not agree quite so well with the analytic result; this is because of the imperfect resolution of our sparse angular grid. However, one must keep in mind that $\lambda_{l=0}$ is in this case almost an order of magnitude smaller than $\lambda_{l=2}$, so the above discrepancy is small compared to the magnitude of $\lambda$. If we increase the number of angular grid zones from 16 to $64, \lambda_{l=0}$ agrees much better with the analytic result, as shown in Fig. 3.

\section{B. Weak-field inhomogeneous collapse I}

We now describe an evolution in which we solve both the field equation (4.1) and the particle equations (2.3) self-consistently. For initial data, it is important not only to choose a configuration that evolves nonspherically, but 

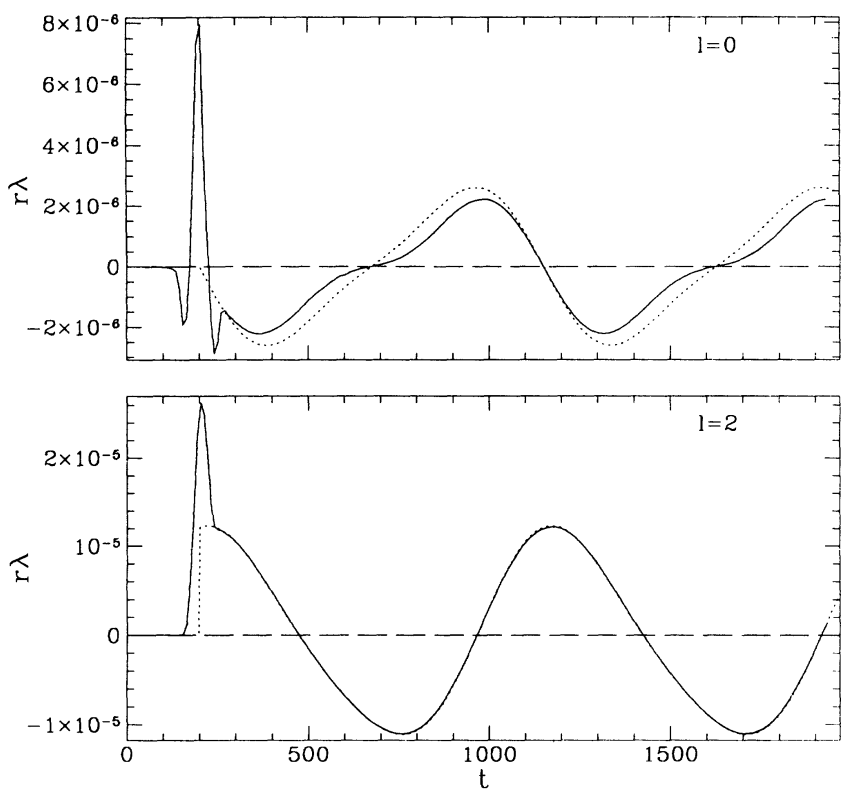

FIG. 2. Wave amplitude $\lambda$ for a case identical to the one shown in Fig. 1, except that the boundary condition imposed at $r=r_{\max }=200 M_{0}$ is Eq. (4.11) rather than Eq. (4.10). Labeling is the same as in Fig. 1. On the bottom plot, the solid and dotted lines are virtually coincident except in the transient region around $t=200 M_{0}$.

also to choose one that will not become singular. We chose an initial matter distribution in the form of an oblate spheroid with $a=50 M_{0}, c=40 M_{0}$, and with an inhomogeneous density profile given by Eq. (5.2). To ensure that the matter does not collapse to a spindle singularity on the axis or a pancake singularity in the equatori-
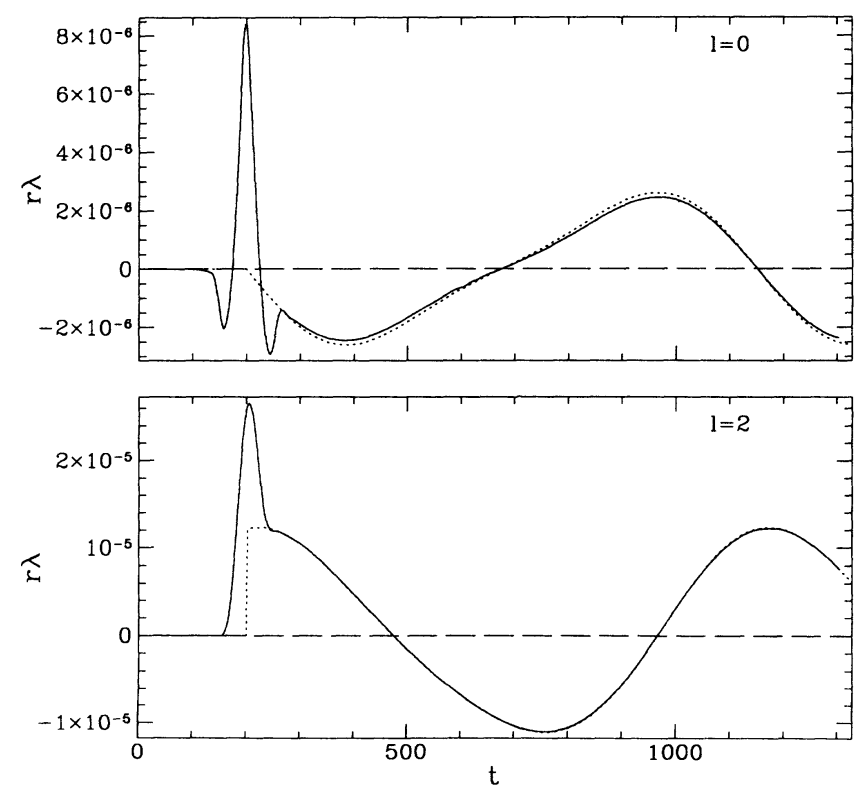

FIG. 3. Wave amplitude $\lambda$ for a case identical to the one shown in Fig. 2, except that the number of angular grid zones has been increased from 16 to 64 . Labeling is the same as in Fig. 1. al plane, we give the particles angular momentum about the axis of symmetry (the $z$ axis) and a velocity dispersion along the axis. We chose initial conditions motivated by the form of the distribution function for Freeman spheroids [9]. Specifically, we define the angular frequencies parallel and perpendicular to the axis by

$$
\begin{aligned}
& \omega_{\|}^{2} \equiv \frac{e^{2 \Phi}}{\tilde{u}^{0}}\left[\frac{\Phi_{, z}}{z}\right]=\frac{e^{2 \Phi}}{\tilde{u}^{0}}\left[\frac{\Phi_{, r}}{r}+\frac{\left(1-x^{2}\right) \Phi_{, x}}{r^{2} x}\right], \\
& \omega_{\perp}^{2} \equiv \frac{1}{r^{2}} e^{2 \Phi}\left[r \Phi_{, r}-x \Phi_{, x}\right] .
\end{aligned}
$$

We then set the initial velocity in the $r_{\perp}$ direction to zero [where $r_{\perp}$ is the radial coordinate in the cylindrical basis $\left.\left(r_{1}, \phi, z\right)\right]$, the conserved angular momentum per unit rest mass to

$$
\tilde{u}_{\phi}=\omega_{1} r^{2} \xi_{\phi}\left(1-x^{2}\right),
$$

and we choose the initial velocity in the $z$ direction according to the distribution

$$
f\left(\widetilde{u}_{z}\right)=\frac{1}{\pi c \omega_{\|}}\left(1-\frac{r^{2}\left(1-x^{2}\right)}{a^{2}}-\frac{r^{2} x^{2}}{c^{2}}-\frac{\widetilde{u}_{z}^{2}}{\omega_{\|}^{2} \xi_{z}^{2} c^{2}}\right)^{-1 / 2} .
$$

Given $\tilde{u}_{z}$, we compute

$$
\begin{aligned}
& \tilde{u}_{r}=x \widetilde{u}_{z}, \\
& \widetilde{u}_{\theta}=-\left(1-x^{2}\right)^{1 / 2} r \tilde{u}_{z} .
\end{aligned}
$$

For a homogeneous spheroid in the weak field limit, $\omega_{\perp}$ and $\omega_{\|}$are constant everywhere inside the matter; in this case, the above velocity distribution with $\xi_{\phi}=\xi_{z}=1$ describes a Freeman spheroid in dynamical equilibrium [9]. Since our density profile is inhomogeneous, $\omega_{1}$ and $\omega_{\|}$depend on both $r$ and $x$, and the resulting configuration is not in dynamical equilibrium, even in the Newtonian limit with $\xi_{\phi}=\xi_{z}=1$.

In order to illustrate that one can obtain reasonably accurate results using modest computer resources, we show our findings obtained using a small number of particles $\left(N_{P}=4000\right)$ and a relatively sparse grid: We use 90 exterior and 90 interior radial grid zones, and 16 angular zones. The outer grid radius is chosen to be $r_{\max }=200$ $M_{0}$. With this choice of parameters, a typical run requires about 0.3 seconds per time step on an IBM Model ES/9000 supercomputer. Approximately two-thirds of this time is spent moving the particles.

Figure 4 shows snapshots of the particle positions at selected times for a run of 10000 time steps in which $\xi_{z}=0.2$ and $\xi_{\phi}=1.0$. The matter distribution clearly evolves nonspherically. The gravitational wave amplitude $\lambda^{\mathrm{rad}}$, measured at a fixed radius $r=87.5 M_{0}$, is shown in Fig. 5. Although the wave amplitude is somewhat noisy, it agrees quite well with the multipole approximation calculated by numerically differentiating Eq. (4.7) with respect to time. We have verified that the wave form becomes smoother as the number of particles $N_{P}$ is increased. 

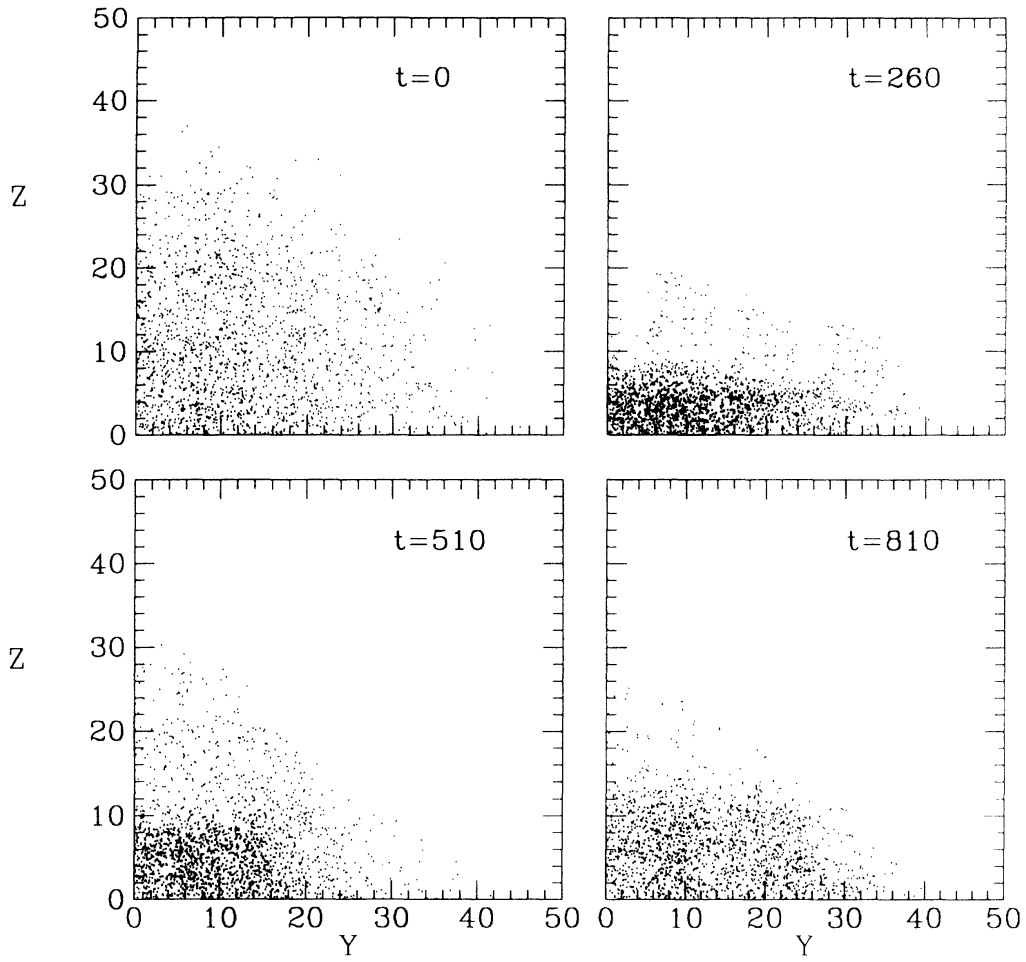

FIG. 4. Snapshots of the particle positions projected into the $y-z$ plane for the evolution of the cluster described in Sec. V B. Particle coordinates and times are in units of $\boldsymbol{M}_{0}$.

\section{Weak-field inhomogeneous collapse II}

It would be interesting to study a system similar to the one in the previous section, but with a cutdown factor $\xi_{\phi}$ significantly less than unity. Such a configuration would collapse to a smaller size than in the previous case, and would thus produce a much larger gravitational wave amplitude. However, if we evolve the system from Sec. V B with a smaller $\xi_{\phi}$, we find that during the initial collapse, the matter distribution develops a sharp surface. This produces a discontinuity in the source term $T$ which prevents us from obtaining smooth waveforms.

We therefore evolve a case with an initial matter distribution identical to the one in the previous section, except that we use an initial $z$-velocity distribution

$$
f\left(\tilde{u}_{z}\right)=\frac{1}{\pi c \omega_{\|}}\left(\frac{r^{2}\left(1-x^{2}\right)}{a^{2}}+\frac{r^{2} x^{2}}{c^{2}}-\frac{\tilde{u}_{z}^{2}}{\omega_{\|}^{2} \xi_{z}^{2} c^{2}}\right]^{-1 / 2},
$$

and we set $\xi_{z}=\xi_{\phi}=0.4$. The velocity distribution (5.9) gives particles near the surface of the spheroid a larger average value of $\tilde{u}_{z}^{2}$ than particles deep in the interior; this tends to prevent the rapid formation of a sharp matter surface. In contrast, the Freeman-like distribution (5.7) requires particles on the surface of the spheroid to have $\tilde{u}_{z}=0$.

Figure 6 shows snapshots of the particle positions at selected times for this case. We see that the evolution is characterized by a rapid initial collapse followed by relaxation towards an equilibrium state in which a centrally condensed region is surrounded by a diffuse nonspherical halo. In Fig. 7 we plot the quantity $-2 K / W$ as a function of time, where $K$ and $W$ are defined in Eq. (2.11).
This quantity is a minimum at $t=0$, when the potential energy dominates, and a maximum at $t=240$, when the configuration is collapsing rapidly. At late times, $-2 K / W$ approaches unity, indicating, according to Eq. (2.12), that the system is approaching virial equilibrium
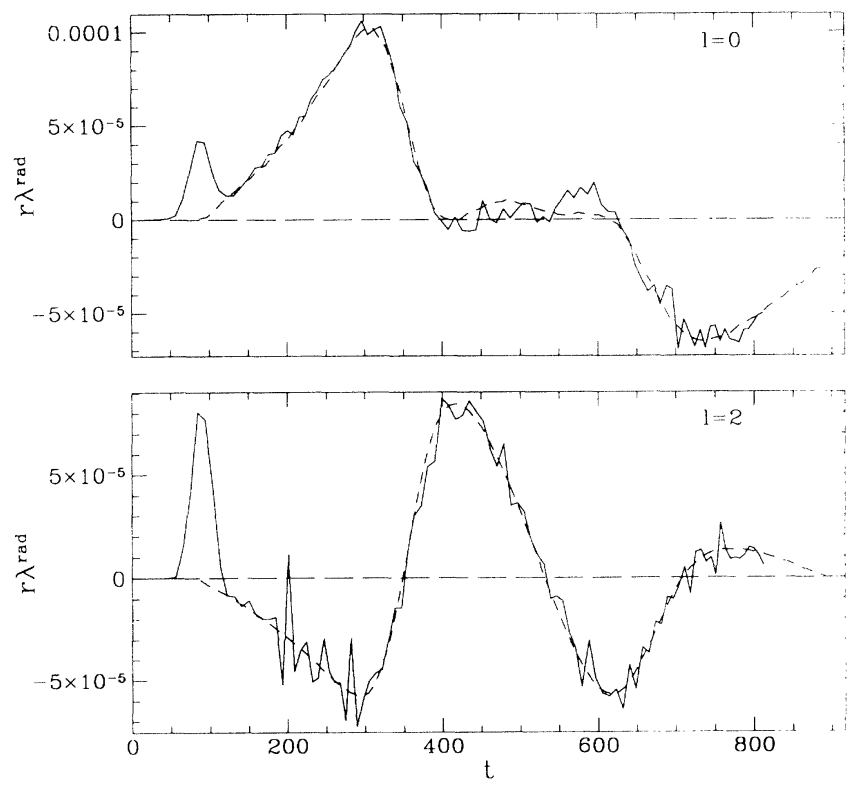

FIG. 5. Gravitational wave amplitude $\lambda^{\text {rad }}$ (multiplied by $r$ ) as a function of time, measured at $r=87.5 M_{0}$, for the evolution shown in Fig. 4. The solid line is the numerical value of $\lambda^{\text {rad }}$ obtained from Eq. (4.1) with outgoing-wave boundary condition (4.11), and the dashed line is the result from the multipole expansion, calculated by taking a numerical time derivative of $\mathrm{Eq}$. (4.7). 

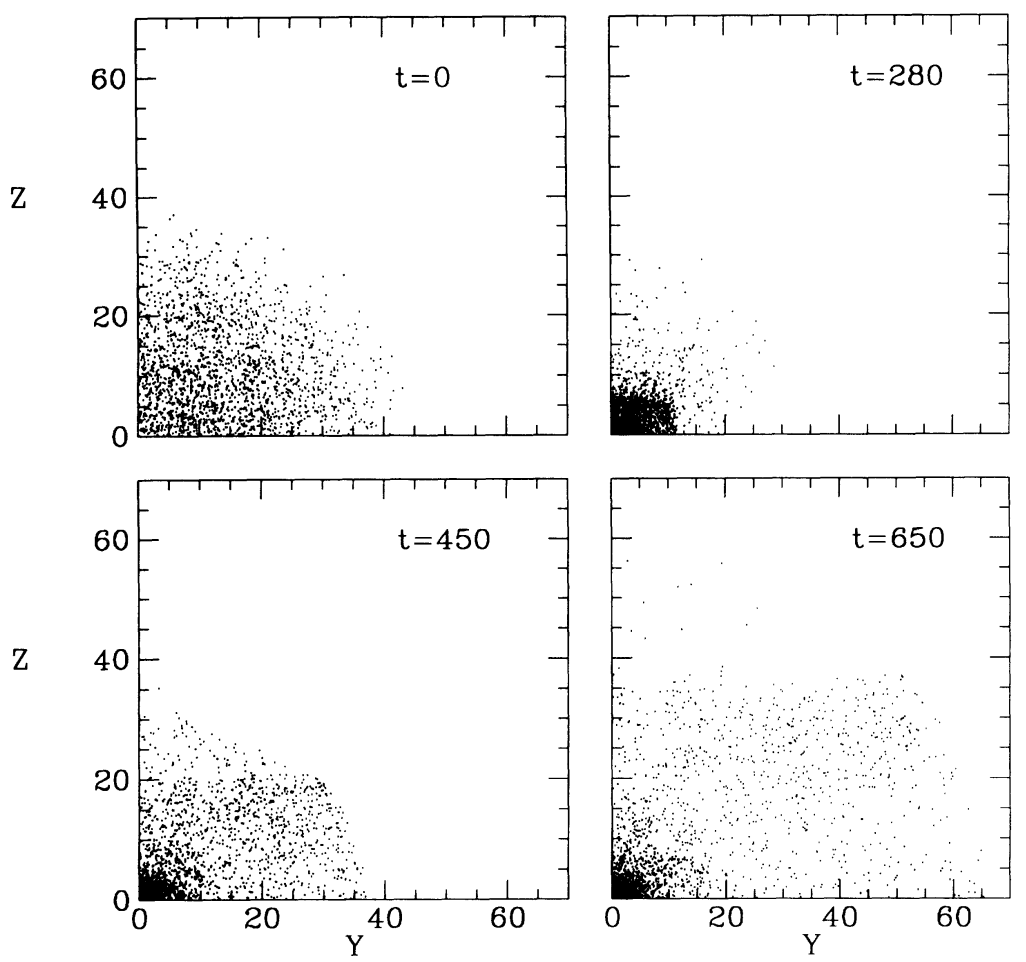

FIG. 6. Snapshots of the particle positions projected into the $y-z$ plane for the evolution of the cluster described in Sec. V C. via violent relaxation.

Because the matter collapses to a smaller configuration in this evolution than in the one discussed in Sec. V B, we expect the wave amplitude to be larger here. This is indeed the case, as shown in Fig. 8. The numerical wave amplitude $\lambda^{\mathrm{rad}}$ agrees well with the multipole expansion formula (4.7). This agreement is good even for the $l=2$

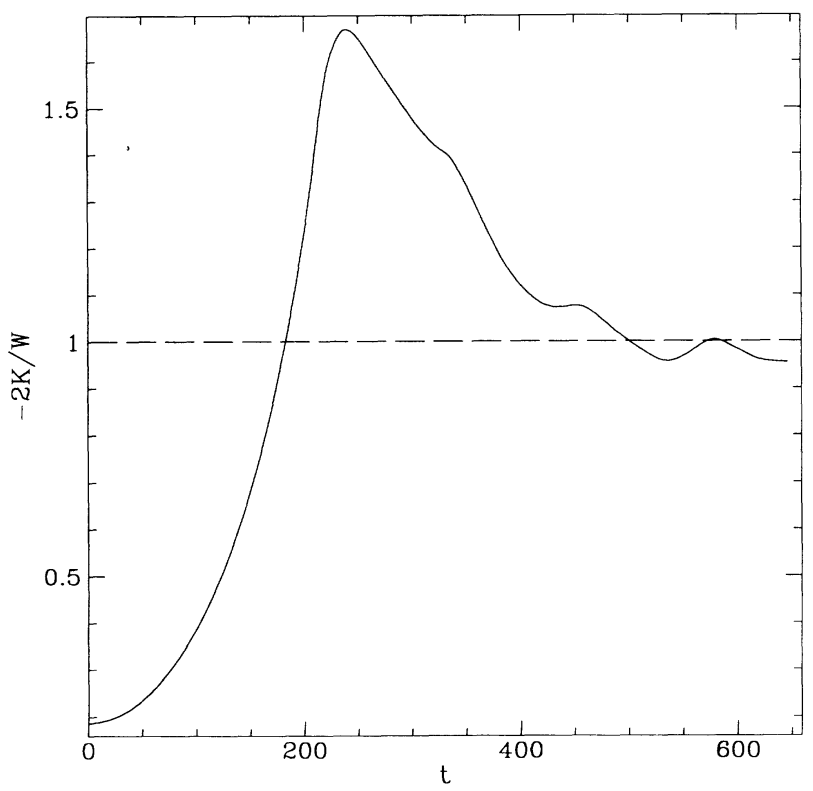

FIG. 7. Approach to virial equilibrium for the evolution shown in Fig. 6, as determined by the special relativistic virial theorem (2.12). Shown is the dimensionless quantity $-2 K / W$ as a function of time. For a system in virial equilibrium, this quantity is equal to unity. component of the wave, which is much smaller than the spherical component.

\section{Strong-field inhomogeneous collapse}

In order to investigate scalar gravitation in the nonlinear strong-field regime, we study the same evolution as in Sec. VB, except that the initial semiaxes of the
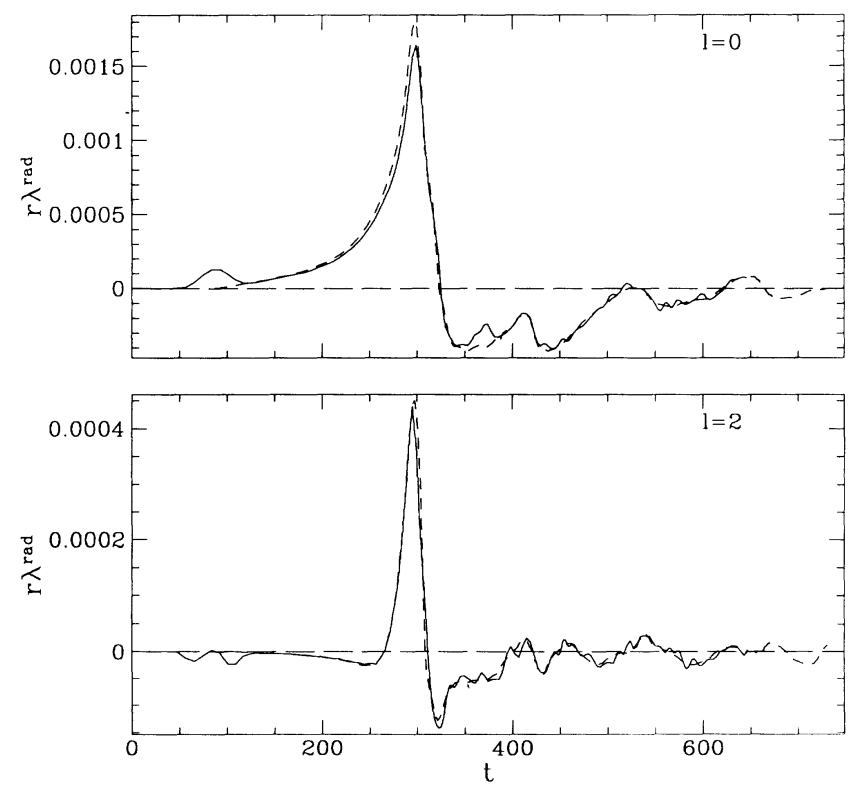

FIG. 8. Gravitational wave amplitude $\lambda^{\text {rad }}$ (multiplied by $r$ ) as a function of time, measured at $r=87.5 M_{0}$, for an evolution shown in Fig. 6. Labeling is the same as in Fig. 5. 

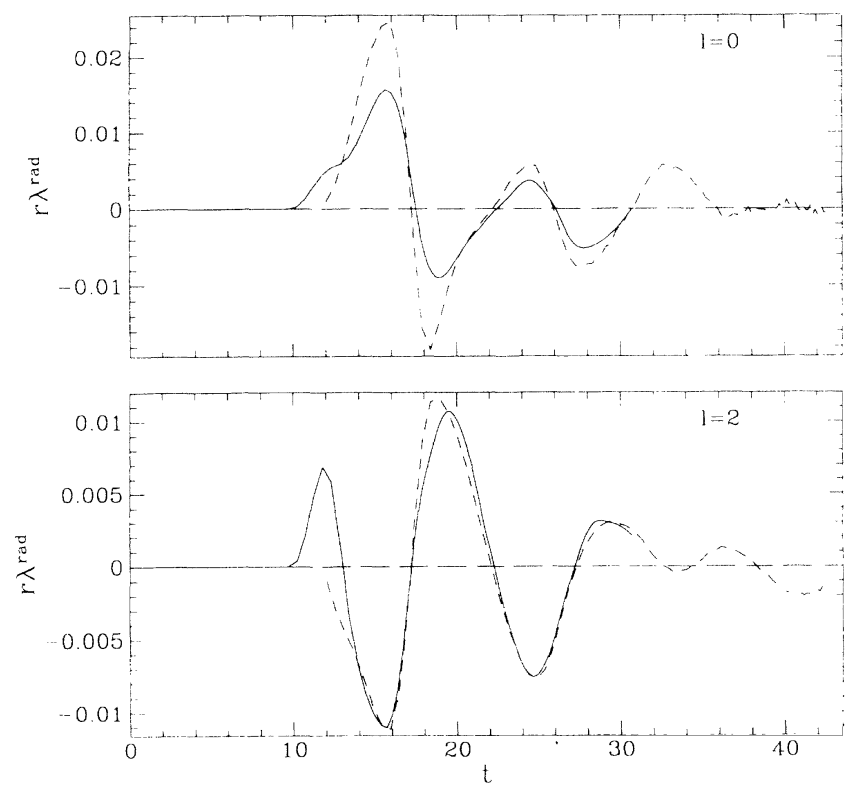

FIG. 9. Gravitational wave amplitude $\lambda^{\text {rad }}$ (multiplied by $r$ ) as a function of time, measured at $r=12 M_{0}$, for an evolution identical to the one shown in Fig. 11, except that the initial size of the configuration has been reduced by a factor of 0.06 to make it relativistic. This evolution required 8000 time steps. Labeling is the same as in Fig. 5.

spheroid are chosen to be $a=3 M_{0}$ and $c=2.4 M_{0}$. The matter evolution is qualitatively similar to the case of Sec. VB: The configuration initially oscillates with a large amplitude parallel to the axis of symmetry, and with a smaller amplitude in the equatorial plane.

The gravitational wave amplitude $\lambda^{\mathrm{rad}}$, as measured at a fixed radius $r=12 M_{0}$, is shown in Fig. 9. Because the gravitational field is strong (initially $\Phi \sim-0.5$ at $r=0$ ), the multipole approximation (3.6) does not agree with $\lambda^{\mathrm{rad}}$. In addition, the disagreement is more pronounced in the $l=0$ component of $\lambda^{\mathrm{rad}}$ than in the $l=2$ component.

Although we no longer have an approximate expression such as the multipole formula (4.7) with which to compare our numerical results, we still can use energy conservation to check the accuracy of our code. In Fig. 10, we use Eq. (2.8) to check the accuracy of our numerical integrations. We see that energy is conserved to within a few percent, even at radii that are partially or completely inside the matter distribution. Both a larger number of particles per grid zone and a denser grid will improve energy conservation. Notice that at small radii, the dashed line in Fig. 10, which represents a quantity $E_{0}(r)=E(r, t=0)$ that is constant in time, actually varies by a small amount. This is because each time the radial grid moves to follow the matter, the function $E_{0}(r)$, as well as all other variables that are defined on the grid, are interpolated from the old grid onto the new one-small errors in this interpolation cause $E_{0}(r)$ to vary slightly in time. This variation becomes negligible as the number of interior radial grid zones is increased.

One of the primary sources of error in Eq. (2.8) associated with having too few particles occurs when the particles are counterstreaming, that is, when particles in the same grid zone are moving in opposite directions. To see why this is so, consider a case in which the velocity distribution is isotropic, and each particle's velocity has the same magnitude. Thus, in the limit of an infinite number of particles, the net flux through any surface is zero. However, suppose that there were only three particles in
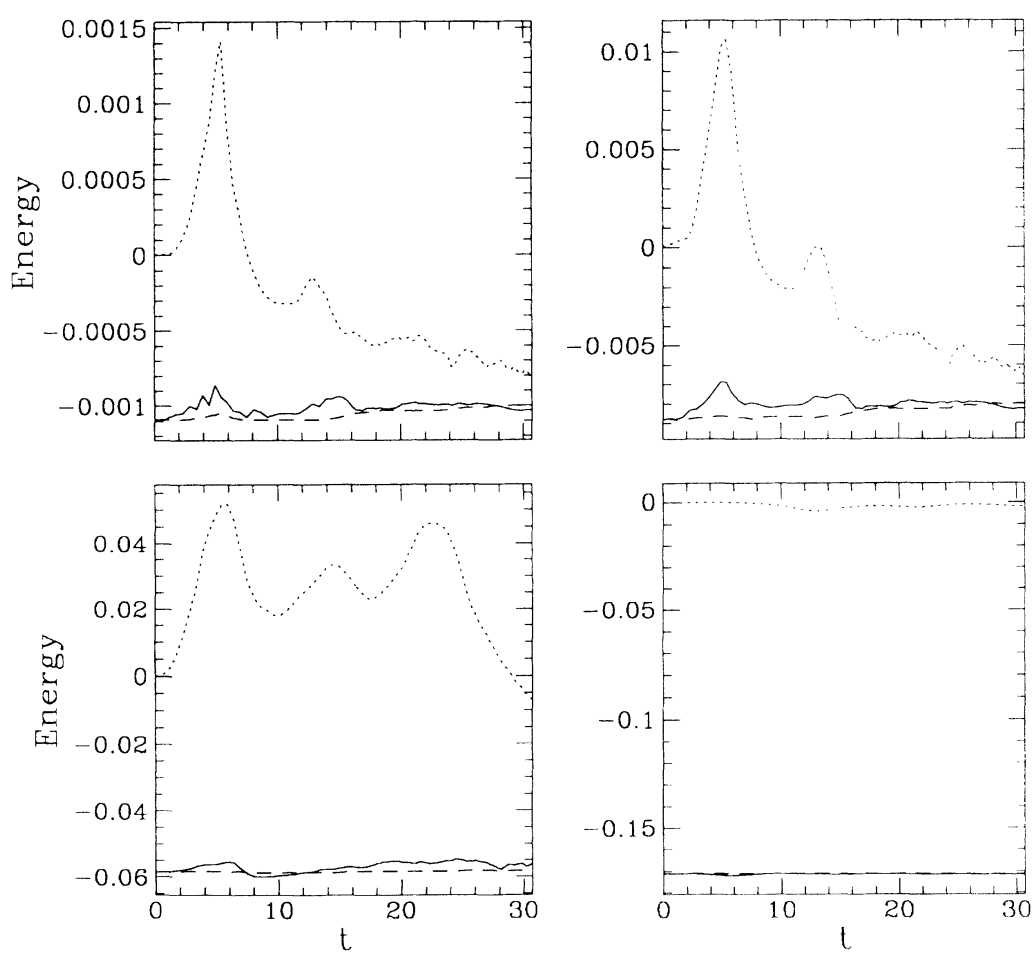

FIG. 10. Energy conservation, Eq. (2.8), at four selected radii as a function of time for the evolution shown in Fig. 9. The dashed line is the initial energy $E(r, t=0)$, the dotted line is the integrated flux $S(r, t)$, and the solid line is the sum of the energy and the flux $E(r, t)+S(r, t)$. Energy and time are in units of $M_{0}$. The radii are, clockwise from upper left, $r=0.25 M_{0}, r=0.5 M_{0}, r=M_{0}$, and $r=7.5 M_{0}$. The degree to which the solid and dashed lines coincide compared with the magnitude of the dotted line is a measure of the code's ability to conserve energy. 
a particular zone: One might find that two are moving radially outward, and the other is moving radially inward, so that the radial flux calculated through that zone would be nonzero. One can always avoid counterstreaming by considering systems that evolve homologously; however, homologous evolution is not likely to occur for any configuration with an inhomogeneous density profile.

\section{vi. CONCLUSIONS}

Although scalar gravitation is not a viable physical theory, it is useful for developing and testing algorithms for numerical relativity. Scalar theory involves fewer field variables than general relativity and is not complicated by black holes or coordinate time singularities, yet it contains many of the generic features of a nonlinear dynamical theory of gravitation, such as gravitational collapse and wave production. We have built a twodimensional mean-field particle simulation scheme for the dynamical evolution of collisionless matter in a selfconsistent scalar gravitational field, and we find that this scheme allows one to calculate smooth and accurate gravitational waveforms, provided that the matter distribution is continuous. By taking advantage of the linearity of the field equation in vacuum, we are able to extract accurately the gravitational wave amplitude from the field variables, and impose a reliable outgoing-wave boundary condition on the scalar field, even at radii not much larger than the matter surface. With sufficient computer resources, a similar mean-field scheme should be able to calculate reliable gravitational wave amplitudes in general relativity.

\section{ACKNOWLEDGMENTS}

We thank A. Abrahams for useful discussions. This research was supported in part by NSF Grants Nos. AST 91-19475 and PHY 90-07834 and NASA Grant No. NAGW-2364 at Cornell University. Computations were performed at the Cornell Center for Theory and Simulation in Science and Engineering, which is supported in part by the National Science Foundation, IBM Corporation, New York State, and the Cornell Research Institute.
[1] C. W. Misner, K. S. Thorne, and J. A. Wheeler, Gravitation (Freeman, San Francisco, 1973).

[2] S. L. Shapiro and S. A. Teukolsky, Phys. Rev. D 47, 1529 (1993).

[3] S. L. Shapiro and S. A. Teukolsky, Phys. Rev. D 48, 592 (1993).

[4] W. L. Burke, J. Math. Phys. 12, 401 (1971).

[5] H. O. Kreiss and J. Oliger, Methods for the Approximate Solution of Time Dependent Problems, GARP Publication Series No. 10 (World Meteorological Organization, Geneva, 1973).
[6] R. W. Hockney and J. W. Eastwood, Computer Simulation Using Particles (McGraw-Hill, New York, 1981).

[7] W. H. Press et al., Numerical Recipes in FORTRAN: the Art of Scientific Computing (Cambridge, New York, 1992).

[8] A. M. Abrahams and C. R. Evans, Phys. Rev. D 37, 318 (1988).

[9] K. C. Freeman, Mon. Not. R. Astron. Soc. 134, 1 (1966); A. M. Fridman and V. L. Polyachenko, Physics of Gravitating Systems (Springer-Verlag, New York, 1984); S. L. Shapiro and S. A. Teukolsky, Astrophys. J. 318, 542 (1987). 

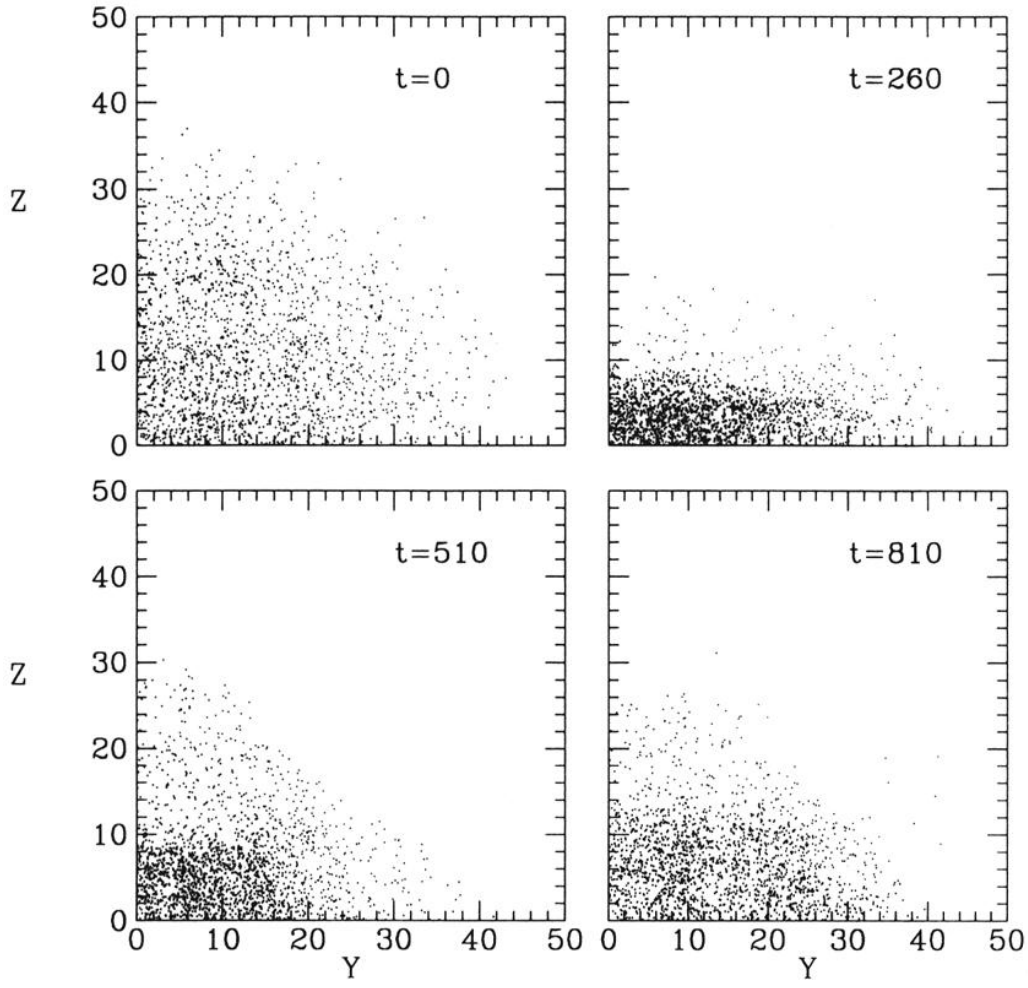

FIG. 4. Snapshots of the particle positions projected into the $y-z$ plane for the evolution of the cluster described in Sec. V B. Particle coordinates and times are in units of $M_{0}$. 

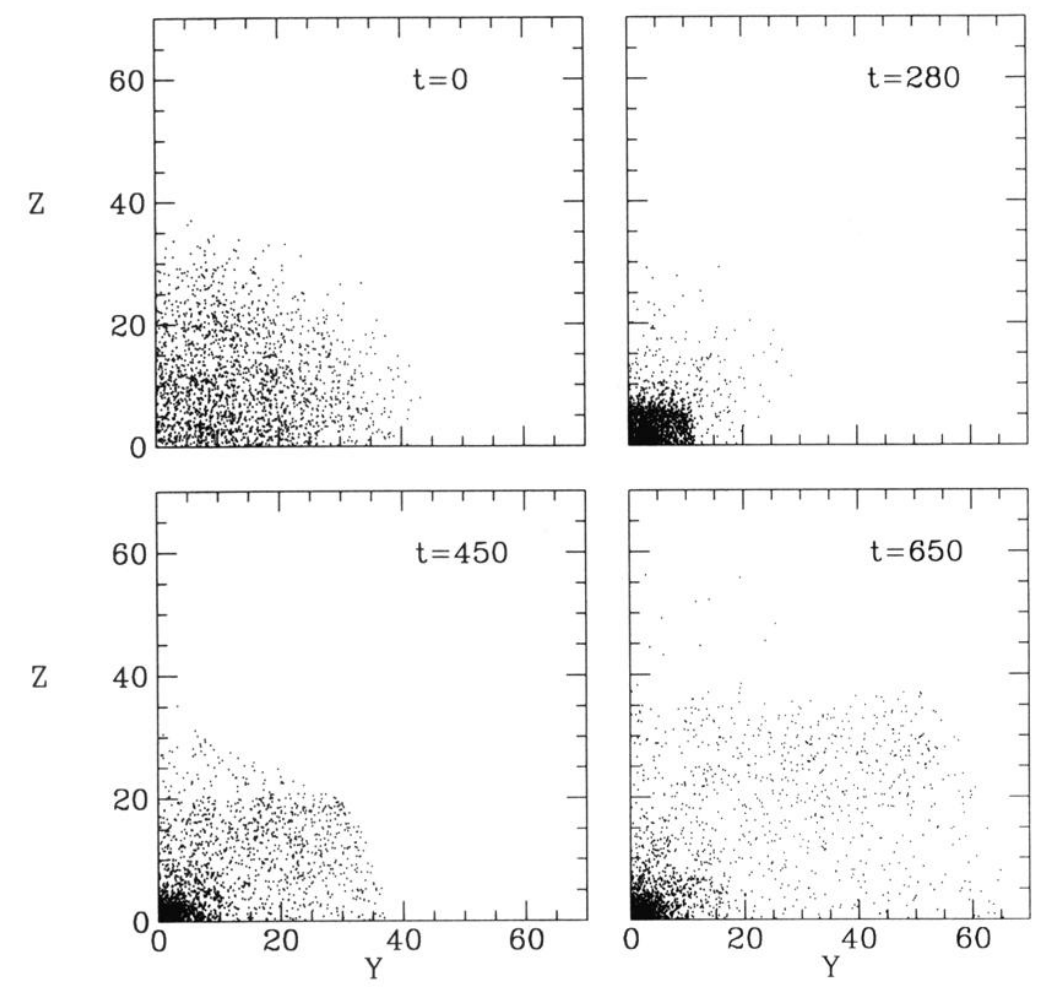

FIG. 6. Snapshots of the particle positions projected into the $y-z$ plane for the evolution of the cluster described in Sec. V C. 